\title{
Adrenal adenocarcinoma with Kartagener's syndrome: A case report
}

\author{
WANLI HU ${ }^{1 *}$, LONG CHENG $^{1 *}$, BEI CHENG $^{2}$, PENG ZHANG $^{1}$, HE XIAO $^{1}$, \\ WENBO WU $^{1}$ and XINGHUAN WANG ${ }^{1}$ \\ ${ }^{1}$ Department of Urology, Zhongnan Hospital; ${ }^{2}$ Department of Human Anatomy, School of Basic Medical Science, \\ Wuhan University, Wuhan, Hubei 430071, P.R. China
}

Received January 29, 2015; Accepted September 14, 2015

DOI: $10.3892 / \mathrm{ol} .2015 .3805$

\begin{abstract}
The present study reports the case of a 44-year-old woman with an adrenal tumor, complicated by Kartagener's syndrome (KS). The patient was admitted to Zhongnan Hospital (Wuhan, China), and presented with an 8-week history of vertigo and extended history of a recurrent cough, accompanied by sputum and a congested nose. Computed tomography indicated a mass on the right adrenal gland and situs inversus. A right adrenal tumor combined with $\mathrm{KS}$ was diagnosed, and resection of the tumor was performed following relief of respiratory symptoms and control of blood pressure. During six months of follow up the patient recovered well from surgery and blood pressure remained stable. This case revealed that patients exhibiting KS may suffer from serious respiratory infections as a result of impaired defense mechanisms against microbes in the airway. Therefore, comprehensive management of infection, safe anesthesia and appropriate surgical procedures for the avoidance of inflammation and trauma are the most significant factors required for the success of the treatment.
\end{abstract}

\section{Introduction}

Kartagener's syndrome (KS) was initially described in 1933 as the classical triad of situs inversus viscerum, chronic sinusitis and bronchiectasis, in a series of four cases reported by Katargener (1,2). Camner et al (3) was the first to suggest primary ciliary dyskinesia (PCD) as the cause of $\mathrm{KS}$ in a report of two patients with $\mathrm{KS}$ who presented ciliary dysfunction and immotile spermatozoa. Afzelius

Correspondence to: Professor Xinghuan Wang, Department of Urology, Zhongnan Hospital, Wuhan University, 169 Donghu Road, Wuhan, Hubei 430071, P.R. China.

E-mail: urologistwxh@gmail.com

*Contributed equally

Key words: adrenal adenocarcinoma, Kartagener's syndrome, situs inversus, retroperitoneoscopy and Eliasson (4) observed ciliary ultrastructural changes resulting from this disease and the absence of dynein arms in the respiratory ciliary axoneme and sperm tail axoneme. According to various studies, KS is included in the group of diseases arising from PCD, an autosomal recessive disorder, and harmful factors in fetal period are etiological factors of PCD and KS (4-7). The prevalence of PCD is estimated to be 1 in 10,000-20,000 and, as 50\% of patients with PCD exhibit situs inversus, the prevalence of $\mathrm{KS}$ is estimated to be 1 in 20,000-40,000. Around 95\% of KS patients are diagnosed before the age of 15 years. There is no significant difference with regard to gender, however, evidence suggests the disease is associated with familial factors and has a tendency towards heredity $(8,9)$.

The present study reports the case of an adrenal adenocarcinoma complicated by KS. To the best of our knowledge, KS has not been previously described in a patient with adrenal adenocarcinoma. The present study discusses the characteristics of $\mathrm{KS}$ in the context of adrenal adenocarcinoma, and reports the comprehensive treatment of the disease in the present case, with the aim of increasing knowledge with regard to the diagnosis and treatment of similar patients.

\section{Case report}

In October 2014, a 44-year-old woman was admitted to Zhongnan Hospital (Wuhan, China) with an 8-week history of vertigo and a recurrent cough, accompanied by sputum and a congested nose. Hypertension (systolic, 140-160 mmHg) was detected by physical examination, and a computed tomography (CT) scan of the abdomen 1 week prior to admission revealed an incidental $5.0 \times 4.0 \mathrm{~cm}$ right adrenal mass, as well as situs inversus. Chest roentgenography and CT scan identified dextrocardia and chronic bronchitis of the lung, with no bronchiectasis (Fig. 1). The patient's blood aldosterone when standing was markedly increased $(308.50 \mathrm{pg} / \mathrm{ml})$, while blood concentrations of potassium, catecholamines and cortisol, as well as urinary excretion of vanilmandelic acid were normal.

The patient exhibited the classical triad of KS (situs inversus, chronic nasosinusitis and bronchiectasia), as well as an underlying adrenal adenocarcinoma. The patient elected to undergo laparoscopic right adrenal tumor resection and was administered phenoxybenzamine $[10 \mathrm{mg}$, 

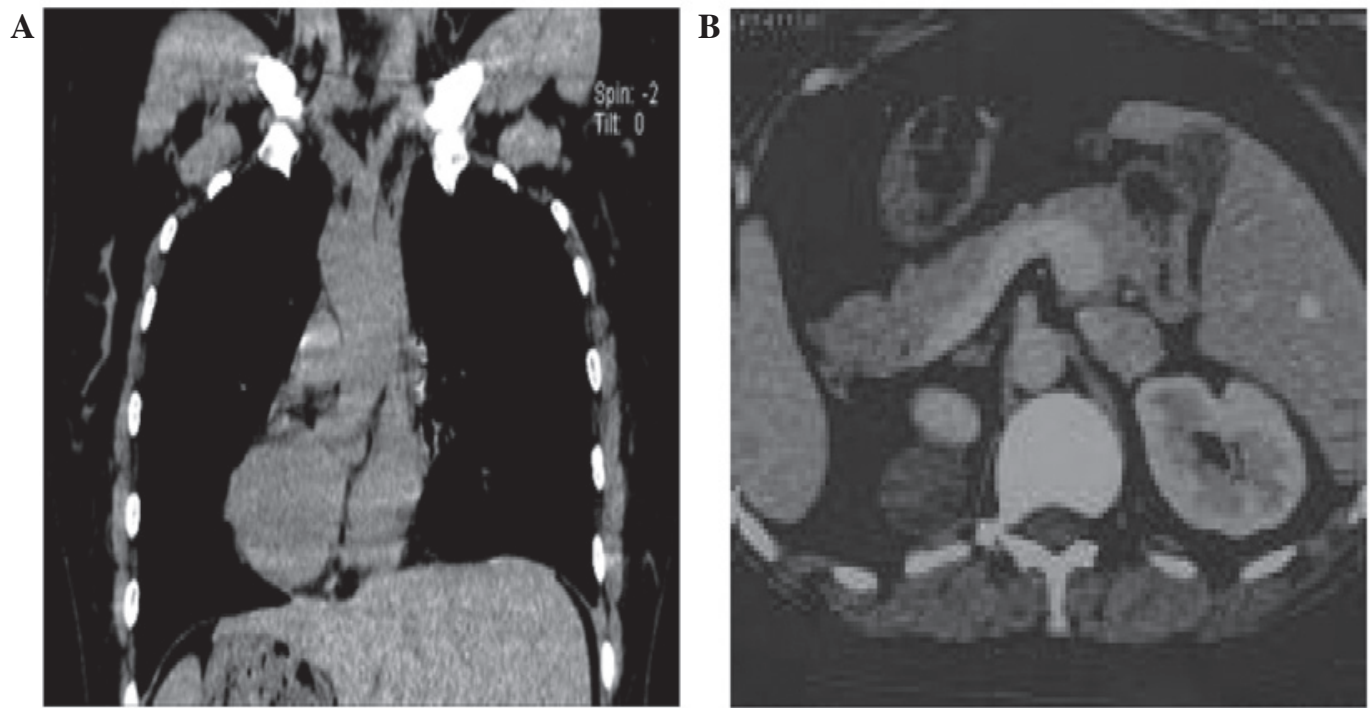

Figure 1. CT scans of the chest and abdomen. (A) Chest CT indicated dextrocardia. (B) Abdomen CT indicated a 5.0x4.0 cm right adrenal mass and situs inversus. CT, computed tomography.
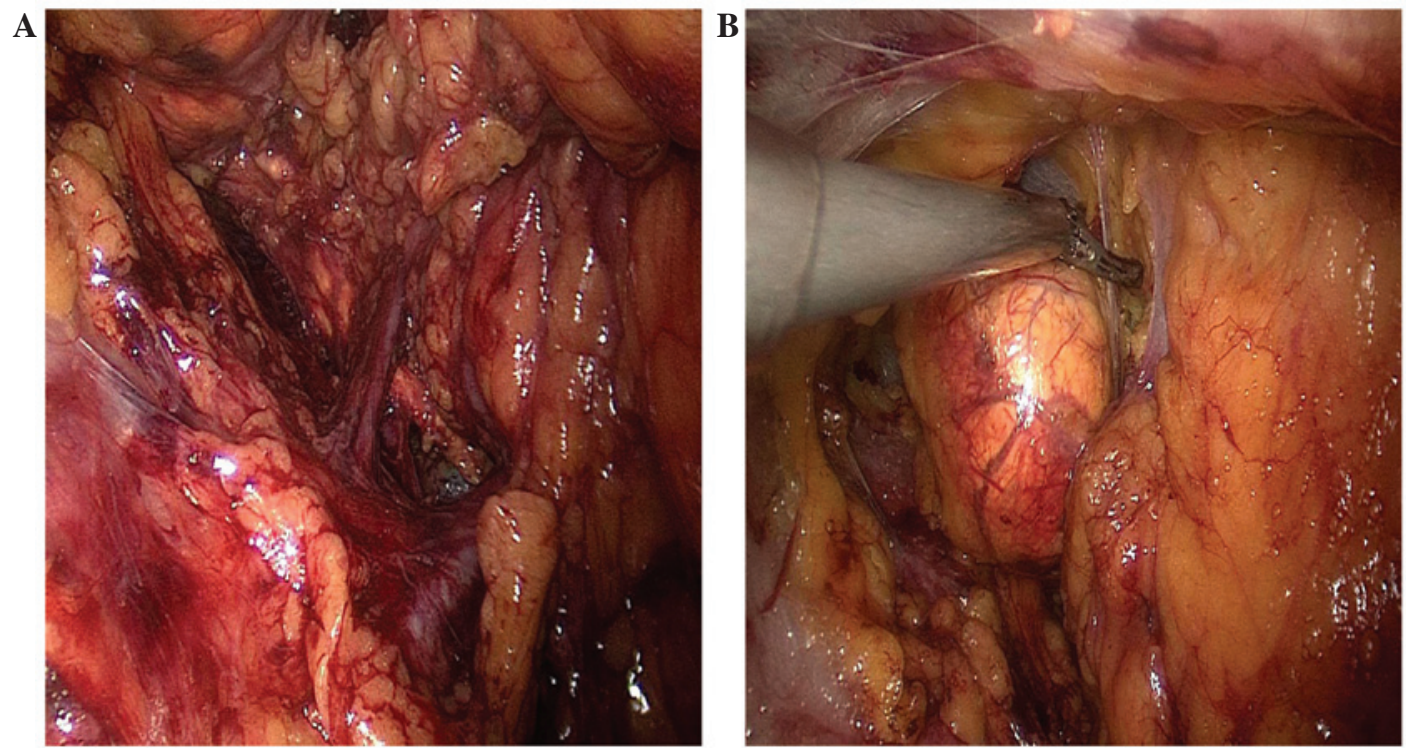

Figure 2. Images captured during surgery. (A) The right adrenal central vein was markedly longer than that of a healthy individual, and flowed into the right renal vein. (B) A harmonic scalpel was used to release the tumor.

three times/day (tid)] and spironolactone (60 $\mathrm{mg}$, tid) orally for one week, to control blood pressure within the range of $120-130 / 80-90 \mathrm{mmHg}$ prior to surgery. Gentamicin (80,000 U), ambroxol hydrochloride (30 mg) and dexamethasone $(5 \mathrm{mg}$ ) were combined and administered as a spray (once daily) to treat the cough and lung infection whilst the patient remained on the waiting list for surgery.

A total of 12 days following admission to hospital, preanesthetic evaluation with all tests had been performed in order to prepare for safe anesthesia and surgery, and the operation was performed using a retroperitoneal laparoscopic approach. Following a preoperative intravenous dose of prophylactic antibiotics (2.5 g, piperacillin-tazobactam), induction of general anesthesia was achieved via administration of intravenous anesthetics ( $2.5 \mathrm{mg}$ midazolam; $80 \mathrm{mg}$ propofol), followed by routine dosage of vecuronium $(6 \mathrm{mg})$ to facilitate endotracheal intubation. Routine monitoring was established, including continuous electrocardiography with mirror image placement of electrodes, pulse oximetry, noninvasive blood pressure monitoring and capnography.

Whilst under general anesthesia, the patient was positioned in a left lateral decubitus. The skin was incised longitudinally, below the twelfth costal arch, in the posterior axillary line. Following blunt dissection of the subcutaneous tissue, the lumbar muscle and fascia were identified and retracted. The retroperitoneum was reached, and an initial digital dissection was performed. Trocars were placed, and visualization of the retroperitoneal space was achieved with the use of $\mathrm{CO}_{2}$ gas. Dissection was performed along the psoas muscle.

During the surgery, the renal hilum vessels were not separated in order to avoid potential injury due to anatomic variations. Anatomical abnormalities were identified in 
relation to the right adrenal gland in the retroperitoneal space. The right adrenal central vein was markedly longer than that of a healthy individual, and was flowing into the right renal vein rather than the inferior vena cava. A harmonic scalpel was used in order to free the superior, lateral, posterior and medial attachments of the adrenal mass (Fig. 2). Following release, the tumor was placed into a bag and held by a grasper. The surgical procedure was performed successfully in $15 \mathrm{~min}$, and the patient's estimated blood loss was $<20 \mathrm{ml}$. Each intraoperative step was accomplished with confidence and no intraoperative complications occurred.

At the conclusion of surgery, the neostigmine (1 mg)-induced neuromuscular blockade was reversed. The patient was extubated and remained in the post-anesthesia care unit for $24 \mathrm{~h}$, where standard care, including electrocardiogram monitoring, $\mathrm{O}_{2}$ inhalation and sputum suction, was administered, and respiratory physiotherapy and exercises were applied in order to clear bronchial secretions and prevent the occurrence of bronchitis. The patient was discharged ten days later and underwent pulmonological and laryngological postoperative follow-up. Pathological analysis of the tumor specimen identified it as an adrenal adenocarcinoma.

\section{Discussion}

$\mathrm{KS}$ is a rare disease, induced as a result of ciliary dysfunction, which occurs due to a defect in the ultrastructure of the cilia (10). The absence of the inner or outer dynein arms impairs the typically coordinated ciliary motion, which leads to mucus retention and subsequently, chronic infections (11). Treatments for KS include the application of antibiotics, cortical hormone or DNase to prevent the progression of complications, including bronchiectasia and chronic nasosinusitis $(12,13)$. Gupta et al (14) emphasized the potential benefits of inoculating patients with flu or pneumococcus vaccines, in order to prevent respiratory tract infections, whilst also performing grommet insertion and endoscopic sinus surgery simultaneously. However, a number of studies have recommended more conservative management of $\mathrm{KS}$, due to the potentially negative effects of surgery $(13,15)$. Overall, the therapeutic goal for $\mathrm{KS}$ is the avoidance of infection and controlling the progression of heart and lung diseases (16).

Particular attention should be paid to imaging studies when patients are diagnosed with adrenal tumors combined with KS, in order to define the size, shape, position and, of particular importance, the surrounding vessels of the tumor. The risks during the perioperative period include: Airway obstruction due to chronic nasosinusitis and bronchiectasia, which may lead to serious respiratory failure; and the difficulty in the elimination of mucopurulent discharge may result in the development of pulmonary edema (17). An appropriate dose of antibiotics, and pulmonary physical therapy, should be administered prior to surgery. Avoiding the use of respiratory inhibitors and center-left trachea cannulas may also reduce the risk of infection (18). In the present study, comprehensive surgical risk assessments were performed and two antibiotics were administered to treat any respiratory symptoms. Laparoscopic retroperitoneal tumor excision was selected as an appropriate surgical strategy, as it generated less trauma, reduced postoperative pain and patient recovery time and presented a reduced risk of pulmonary complications (19). Surgical interventions in KS patients are more difficult due to potentiality complex anatomical variations (20). Oms et al (21) reported the advantages of using left-handed surgeons to perform cholecystectomy for patients exhibiting $\mathrm{KS}$, indicating that it may be useful to alter standard operating procedures to meet the demands of such anatomical variations. Laparoscopic adrenal adenocarcinoma excisions are typically performed by a transabdominal pre-peritoneal approach for removal of abdominal organs and to allow sufficient operating space, however this type of surgery affects the internal environment of the abdominal cavity (22) Therefore, in the present study, the retroperitoneal route was selected in order to reach the right adrenal gland in the shortest feasible distance, and thus improve the safety of the surgery.

In conclusion, in the present study, the feasibility of adrenal tumor resection via a retroperitoneal approach, using laparoscopic instruments, was confirmed. Prevention and treatment of respiratory infection, as well as carefully observing anatomical variations during surgery is of particular importance for patients exhibiting adrenal tumors combined with KS. In this manner, an ideal balance between safety and effectiveness is achievable.

\section{References}

1. Berdon WE and Willi U: Situs inversus, bronchiectasis, and sinusitis and its relation to immotile cilia: History of the diseases and their discoverers - Manes Kartagener and Bjorn Afzelius. Pediatr Radiol 34: 38-42, 2004.

2. Kartagener M: Zur Pathogenese der Bronchiektasien. Bronchiektasien bei Situs inversus viscerum. Beitr Klin Tuberk 83: 489-501, 1933 (In German). doi: 10.1007/BF02141468.

3. Camner P, Mossberg B and Afzelius BA: Evidence of congenitally nonfunctioning cilia in the tracheobronchial tract in two subjects. Am Rev Respir Dis 112: 807-809, 1975.

4. Afzelius BA and Eliasson R: Male and female infertility problems in the immotile-cilia syndrome. Eur J Respir Dis Suppl 127:144-147, 1983.

5. Eliasson R, Mossberg B, Camner P and Afzelius BA: The immotile-cilia syndrome. A congenital ciliary abnormality as an etiologic factor in chronic airway infections and male sterility. N Engl J Med 297: 1-6, 1977.

6. Armengot Carceller M, Carda Batalla C, Escribano A and Samper GJ: Study of mucociliary transport and nasal ciliary ultrastructure in patients with Kartagener's syndrome. Arch Bronconeumol 4: 11-15, 2005 (In Spanish).

7. Geremek M and Witt M: Primary ciliary dyskinesia: Genes, candidate genes and chromosomal regions. J Appl Genet 45: 347-361, 2004.

8. Afzelius BA and Stenram U: Prevalence and genetics of immotile-cilia syndrome and left-handedness. Int J Dev Biol 50: 571-573, 2006.

9. Cowan MJ, Gladwin MT and Shelhamer JH: Disorders of ciliary motility. Am J Med Sci 321: 3-10, 2001.

10. Ceccaldi PF, Carré-Pigeon F, Youinou Y, et al: Kartagener's syndrome and infertility: Observation, diagnosis and treatment. J Gynecol Obstet Biol Reprod (Paris) 33: 192-194, 2004 (In French).

11. Dhar DK, Ganguly KC, Alam S, Hossain A, Sarker UK, Das BK and Haque MJ: Kartagener's Syndrome. Mymensingh Med J 18: 75-79, 2009.

12. Flume PA, O'Sullivan BP, Robinson KA, et al; Cystic Fibrosis Foundation, Pulmonary Therapies Committee: Cystic fibrosis pulmonary guidelines: Chronic medications for maintenance of lung health. Am J Respir Crit Care Med 176: 957-969, 2007.

13. Desai M, Weller PH and Spencer DA: Clinical benefit from nebulized human recombinant DNase in Kartagener's syndrome. Pediatr Pulmonol 20: 307-308, 1995.

14. Gupta S, Handa KK, Kasliwal RR and Bajpai P: A case of Kartagener's syndrome: Importance of early diagnosis and treatment. Indian J Hum Genet 18: 263-267, 2012. 
15. McManus IC, Mitchison HM, Chung EM, Stubbings GF and Martin N: Primary ciliary dyskinesia (Siewert's/Kartagener's syndrome): Respiratory symptoms and psycho-social impact. BMC Pulm Med 3: 4, 2003

16. D'Auria E, Palazzo S, Argirò S, El OS and Riva E: Primary ciliary dyskinesia: Clinical and genetic aspects. Pediatr Med Chir 34: 117-122, 2012 (In Italian).

17. Savitha KS, Sunanda S and Vijayan R: Kartagener's syndrome Anaesthetic implications. Indian J Anaesth 50: 469-471, 2006.

18. Kashif A, Masud M, Manzoor SM and Haneef S: Kartagener's syndrome and acute appendicitis. J Ayub Med Coll 22: 176-177, 2010.

19. Hazzan D, Shiloni E, Golijanin D, et al: Laparoscopic vs open adrenalectomy for benign adrenal neoplasm. Surg Endosc 15: $1356-1358,2001$
20. Biere SS, van Berge Henegouwen MI, Maas KW, Bonavina L, Rosman C, Garcia JR, Gisbertz SS, Klinkenbijl JH, Hollmann MW, de Lange ES, et al: Minimally invasive versus open oesophagectomy for patients with oesophageal cancer: A multicentre, open-label, randomised controlled trial. Lancet 379: 1887-1892, 2012.

21. Oms LM and Badia JM: Laparoscopic cholecystectomy in situs inversus totalis: The importance of being left-handed. Surg Endosc 17: 1859-1861, 2003.

22. Buell JF, Abreu SC, Hanaway MJ, et al: Right donor nephrectomy: A comparison of hand-assisted transperitoneal and retroperitoneal laparoscopic approaches. Transplantation 77: 521-525, 2004. 